\title{
Amusing ourselves to death? Superstimuli and the evolutionary social sciences
}

\author{
Author version (of final peer reviewed manuscript) \\ To appear in: 23(6) (2010) Philosophical Psychology 821-843
}

Andreas De Block \& Bart Du Laing

\begin{abstract}
Some evolutionary psychologists claim that humans are good at creating superstimuli, and that many pleasure technologies are detrimental to our reproductive fitness. Most of the evolutionary psychological literature makes use of some version of Lorenz and Tinbergen's largely embryonic conceptual framework to make sense of supernormal stimulation and bias exploitation in humans. However, the early ethological concept "superstimulus" was intimately connected to other erstwhile core ethological notions, such as the innate releasing mechanism, sign stimuli and the fixed action pattern, notions that nowadays have, for the most part, been discarded by ethologists. The purpose of this paper is twofold. First, we will reconnect the discussion of superstimuli in humans with more recent theoretical ethological literature on stimulus selection and supernormal stimulation. This will allow for a reconceptualisation of evolutionary psychology's formulation of (supernormal) stimulus selection in terms of domain-specificity and modularity. Second, we will argue that bias exploitation in a cultural species differs substantially from bias exploitation in non-cultural animals. We will explore several of those differences, and explicate why they put important constraints on the use of the superstimulus concept in the evolutionary social sciences.
\end{abstract}

\section{Keywords}

Superstimulus, theoretical ethology, evolutionary psychology, bias exploitation, cultural evolution

\begin{abstract}
Affiliations
Andreas De Block is an Assistant Professor at the Institute of Philosophy, University of Leuven, Belgium.

Bart Du Laing is a Postdoctoral Fellow of the Research Foundation - Flanders (FWO) at the Department of Legal Theory and Legal History, Ghent University, Belgium.

Correspondence to: Andreas De Block, Institute of Philosophy, University of Leuven, Kardinaal Mercierplein 2, 3000 Leuven, Belgium. Email: andreas.deblock@ hiw.kuleuven.be
\end{abstract}

\section{Acknowledgements}

The authors wish to thank Arnold Burms, Wouter D'Hooghe, Stefano Ghirlanda, Darian Meacham, and two anonymous referees for their helpful and inspiring comments. As regards the second author, this work was supported by the Research Foundation - Flanders (FWO). 


\section{Introduction}

In 2006, the Edge Foundation asked several people in the arts and sciences for a short piece about their "dangerous idea". In his contribution, the evolutionary psychologist Geoffrey Miller suggested an original solution for the Fermi paradox: the contradiction between the high probability of the existence of extraterrestrial intelligence and the lack of contact with civilized aliens. Miller postulated that it is quite likely that intelligent aliens do not come and visit us because they are just too busy having fun with computer games and pornography. In that respect, they are just like us. Miller writes:

I suspect that a certain period of fitness-faking narcissism is inevitable after any intelligent life evolves. This is the Great Temptation for any technological species to shape their subjective reality to provide the cues of survival and reproductive success without the substance. Most bright alien species probably go extinct gradually, allocating more time and resources to their pleasures, and less to their children. (Miller 2006)

Maybe Miller's idea is a dangerous one. The kernel of the idea, however, is not new. Many evolutionary psychologists and, before them, (human) ethologists have developed a similar line of reasoning. Steven Pinker, for instance, argues that a great deal of human culture, including most of the arts, can be seen as what he calls "cheesecake for the mind". In his view, the arts, pornography and strawberry cheesecake are all superstimuli:

We enjoy strawberry cheesecake, but not because we evolved a taste for it. We evolved circuits that gave us trickles of enjoyment from the sweet taste of ripe fruit, the creamy mouth feel of fats and oils from nuts and meat, and the coolness of fresh water. Cheesecake packs a sensual wallop unlike anything in the natural world because it is a brew of megadoses of agreeable stimuli which we concocted for the express purpose of pressing our pleasure buttons. (Pinker 1997, 524)

Both Pinker and Miller claim that humans are good at creating superstimuli, and that such pleasure technologies are detrimental to our reproductive fitness. And while at least a part of 
their claim seems plausible, many problems lurk behind the use of the superstimulus-idea in the evolutionary social sciences. This paper will criticize the use of the superstimulus-idea in evolutionary psychology and related disciplines. We do not deny that the superstimulusconcept can substantially enrich our understanding of human behavior. However, a fruitful and scientifically warranted application requires both (1) more conceptual clarity and detail than is currently available, and (2) acknowledging that our species is profoundly cultural. The first section looks at the development of the superstimulus-idea. The second section discusses and criticizes the use of superstimulus-theory in evolutionary psychology. In the third section, we construct a detailed, but nevertheless workable, taxonomy of superstimuli for the evolutionary social sciences, solving at least some of the problems associated with the evolutionary psychology approach. The fourth section explores to what extent humans and other profoundly cultural beings from elsewhere in the universe are vulnerable to exploitation by superstimuli.

\section{The Tinbergen experiment and its interpretations}

Early theories of ethology focused on fixed action patterns (FAPs). A FAP was defined as an indivisible sequence of innate behaviours that (1) can be produced in all normal individuals of a single species, and (2) once started cannot be stopped until the entire action sequence is completed. Lorenz saw FAPs as unlearned actions activated by innate releasing mechanisms (IRMs) that were thought to occur in response to key stimuli.

Animals not only react to key stimuli, but also to modifications of key stimuli. Usually, modifications lead to a weaker response, but certain new stimuli can cause an increase responding. In a series of now classical experiments, Tinbergen discovered that exaggerated versions of key stimuli sometimes produce stronger reactions than the natural key stimuli.

In the early 1920s, Oskar Heinroth, the father of comparative behavioral biology, had already observed that Herring Gull chicks often peck at a variety of red objects (Tinbergen 1948). According to Tinbergen, the redness of these objects was a key stimulus, stimulating a begging response in the Herring Gull chick. He correlated the red spot on the Herring Gull's yellow bill with the chick's feeding. In general, the outcome of the pecking at the contrasted spot is that the chick finds the food in the parent's bill. However, in some cases, for instance when the red object is a rubber shoe sole, pecking at red objects does not result in finding 
food. With his student Ab Perdeck, Tinbergen set up several experiments to test the hypothesis that objects similar to the key stimulus could activate the FAP. They presented chicks with various painted carton dummies of a Herring Gull's head. Every time a model was presented, a student simulated the call normally given by a parent-bird. The dummy was then held in front of the chick for thirty seconds, and the number of pecking reactions counted.

One model was a three-dimensional, accurately shaped and colored model of the parent-gull's head and beak. Another was an accurate model of the bill only. A third model was a thin red rod with three sharply edged white bands at its tip, a very inaccurate representation of the adult gull's head. The rod contained more red, more color contrast, and was much more elongated. Nevertheless, this highly artificial stimulus (stimuli) received about $25 \%$ more pecks from the gull chicks than the other models (and the natural head). Tinbergen called the rod a superstimulus (Tinbergen \& Perdeck 1950).

Tinbergen was not particularly happy with the results of this experiment (Kruuk 2003). He implicitly assumed that the behavior of animals is highly adaptive. The fact that the chick exhibited a stronger response to an artificial stimulus than to the natural key stimulus seemed to contradict that assumption. Yet, instead of changing his assumptions about the adaptive value of all animal behaviour, Tinbergen tried to explain away the result by claiming that the elongated dummy was in fact an accurate representation of what the chick saw in its natural environment (Tinbergen 1951). Tinbergen's questionable interpretation of the experiment was not followed by other theoreticians (Hailman 1961). Rather, most other ethologists interpreted this experiment as showing high discrimination for certain key properties of the original stimulus to be more important for the activation of the releasing mechanism than fidelity to the original stimulus. This was not only true for the pecking response in the Herring Gull chicks, but also applied to many other responses in many other species. Magnus (1958) reported that an extra large size of the mate model increases male response in the butterfly Argynnis paphia. When an oversized artificial egg was placed into the nests of Oystercatchers, these birds preferred it to a normal sized egg, even though they were unable to brood the artificial egg because of its large size (Tinbergen 1951).

Why do some animals respond more to a superstimulus that neither they nor their forbearers have ever seen than to the natural stimuli? Several answers have been given to this question. 
They can be split up into two (rough) categories: some have argued that (1) the reaction is a learning effect; others hold that (2) the tendency to react stronger to more discriminative features is innate. Among the learning-effect explanations, the peak shift (Hansson 1959) is certainly the best known and most popular in the scientific literature. The peak shift is a displacement in the mode of a post-discrimination stimulus generalization gradient away from its expected location. When an animal has learned to distinguish between a positive stimulus (a stimulus correlated with reinforcement) and a negative stimulus (a stimulus correlated with extinction) lying on the same stimulus dimension, the animal will maximally respond in a new experiment, not to the original positive stimulus, but to a stimulus further from the negative stimulus. Peak shifts occur when the discrimination is learned with errors, making the negative stimulus an aversive one. In such cases, the peak shift is a shift away from an aversive stimulus (Terrace 1966). In the innate category, almost all explanations tend to see the stronger response to superstimuli as the effect of recognition system biases. Such biases may be adaptive. Engelmann (1970) suggests that the preference for certain superoptimal stimuli enhance male and female mate orientation in some insects, for example, the butterfly Argynnis paphia. However, even if the innate perception biases are generally adaptive in the natural environment (e.g., when the biggest mates are usually the fittest ones) that does not mean that these biases cannot be exploited. Nearly every experiment on superstimuli has done just that. Even in the selective environment, exploitation of the biases occurs. Many animals produce superstimuli to manipulate the signal-receiver in such a way that the response of the signal-receiver is detrimental for the inclusive fitness of the signal-receiver and beneficial for the signal-sender's inclusive fitness (Dawkins \& Krebs 1978). Lack notes, for example, that the cuckoo chick

with its huge gape and loud begging call, has evidently evolved in exaggerated form the stimuli which elicit the feeding response of parent passerine birds. [...] This, like lipstick in the courtship of mankind, demonstrates successful exploitation by means of a "super-stimulus". (Lack 1968, as quoted by Atran 2006, 306)

As far as Tinbergen's findings are concerned, the second innate option seems closest to his thinking. He shared Lorenz's conviction that FAPs were triggered by innate releasing mechanisms. They both held that the innate releasing mechanisms remained largely unmodified by experience during development (Tinbergen 1951). 


\section{Superstimuli and maladaptive behavior in evolutionary psychology}

Tinbergen's theory of superstimuli is widely known in the community of behavioral ecologists, but only rarely used. Some evolutionary social scientists, however, do invoke superstimuli quite often to explain human behaviour. This is especially true for evolutionary psychologists. Their fondness for the theory probably has two intimately intertwined explanations. First, they are interested in maladaptive behavior. Inasmuch as, (a) the superstimuli differ from the original key stimuli, and (b) the responses to the superstimuli are exaggerated, superstimuli form a source of maladaptive behavior. Other evolutionary social sciences, like human behavioral ecology, tend to be less interested in maladaptive behavior, which may explain why they ignore the superstimulus-theory altogether. Second, evolutionary psychologists see the cultural environment as the factor responsible for many if not most of our species's maladaptive thinking and behavior. Because the superstimuli Tinbergen and his collaborators used were artifacts (the stick, the plaster egg, etc.), evolutionary psychologists seem to think that Tinbergen's experiments may be one of the keys to a deeper understanding of our own maladaptive reactions to artifacts. Dual inheritance theorists share the evolutionary psychological view that maladaptive behavior should be an important target of evolutionary approaches to human behavior, but since they think that cultural transmission biases - and not the cultural environment as such - are the prime culprits for human maladaptive behavior, superstimuli are almost completely absent from their explanatory framework.

Before we further investigate the actual use of the superstimulus-idea in evolutionary psychology, we have to point out that no evolutionary psychologist actually thinks that superstimuli are the only factors causing maladaptive behavior. In evolutionary psychology, the encompassing explanatory model for widespread maladaptive behavior is a mismatchmodel. Evolutionary psychologists claim that the "Environment of Evolutionary Adaptedness" (EEA) differs substantially from the modern cultural environment. As a result, we are much better at solving the sort of problems our hunter-gatherer ancestors faced than the problems we encounter in modern cities. This mismatch model consists of three submodels or hypotheses. First, our modern environment probably frustrates many of our deep-seated evolved needs: "If we are to understand the psychiatric disorders from which our contemporaries suffer, then we have to take into account the ways in which Western society frustrates the needs of paleolithic men or women still persisting as living potential within us in our present environmental circumstances." (Stevens \& Price 2000, 35). Second, many of 
the adaptive problems that we currently face were not adaptive problems on the East African savannahs in the Pleistocene. ${ }^{1}$ We do not fear guns the way we fear snakes, even though guns pose a much greater threat to our fitness today than snakes do (Öhman \& Mineka 2001, cf. also Blanchette 2006). Third, some objects or events in our cultural environment resemble objects or events from our ancestors' environment. These modern objects or events trigger the reactions that evolved as adaptive responses to the Pleistocene objects and events. The problem is that the new entities also differ from the old entities in some respects. These differences mean that the same reaction that was adaptive in the Pleistocene is now maladaptive.

Obviously, the superstimulus-theory is especially important for the third claim. Moreover, the third claim may be identical to the idea that humans are not immune to the powers of superstimuli. To decide whether or not this is the case, one must review the ways the theory has been applied to humans.

Superstimuli are most often invoked to explain our interest in art or art-like objects. Already in the 1960s and 1970s, several art theorists adopted the notions of superstimulus and supernormal releasers to account for the phenomenon of aesthetic excitement (see, e.g., Coss 1968, Behrens \& Whitson 1976). Contemporary evolutionary psychologists place themselves in this tradition when they claim that visual arts, music, and literature are for humans what big plaster eggs are for geese and elongated sticks with red dots are for Herring Gulls. Ramachandran has put it this way:

For instance, a gull chick will beg for food by pecking at the red spot on its mother's long yellow beak. Niko Tinbergen found that a long stick with three red stripes on it is, paradoxically, much more effective at stimulating fervent begging than a beak, even though it does not resemble one. Such ultranormal stimuli must excite beak-detecting visual neurons in the chick's brain more powerfully than an actual beak does, because of certain accidental features of these neurons' wiring (perhaps embodying the rule "the more red contour the better"). So if gulls had art galleries, they might hang this abstract pattern on the wall, worship it, pay millions of dollars for it (even call it a

\footnotetext{
${ }^{1}$ Human behavioural ecologists tend to be very sceptical about the three submodels, but are especially critical about the claim that humans are unable to solve new problems because they are prisoners of their evolved adaptations to past environment (Irons 1998).
} 
Picasso), yet not understand why - given that the strange pattern doesn't even resemble anything. I would argue that the same situation holds for nonrealistic or semi-abstract art that we humans enjoy. (Ramachandran 2004, 780)

In much the same way, the neurobiologist Semir Zeki suggested that Mondrian's highly abstract paintings exploit the perceptual processes in certain areas of the visual cortex, eventually leading to an evaluation of beauty (Zeki 1999). According to others, the same reasoning may hold for literature and music. The musicologist Knobloch believes that music causes pleasurable sensations, partly because it imitates evolutionary programmed releasers such as laughter and human voices, "possibly as supernormal stimuli” (Knobloch 1995/2000). In general, Steven Pinker endorses this idea, although he seems to be rather reluctant with regard to the examples given by Zeki and Ramachandran. Modern and postmodern art is clearly not his cup of tea: Pinker interprets the sneering of contemporary artists at the bourgeoisie as "a sophomoric grab at status with no claim to moral or political virtue." Twentieth century art is, in his view, the result of "a militant denial of human nature. Our legacy is ugly, baffling, and insulting art." (Pinker 2003, 416) However, real art, i.e., what qualifies as beautiful art in Pinker's view, is most probably a matter of code breaking: real artists intuitively know what the human pleasure buttons are, and they master the techniques to paint, compose or write down the material necessary to push these buttons even harder than the naturally occurring key stimuli.

Of course, the use of superstimuli-theory in the evolutionary social sciences is not restricted to art. Almost all aspects of culture have been linked to superstimuli. In the early (and highly speculative) days of human sociobiology, Desmond Morris mentioned gymnastics, beds ("supernormal beds"), perfume, and even the sporran (furry wallet) of the Scottish kilt as supernormal stimuli (Morris 1969). Nowadays, relying on superstimuli-theory is a common strategy if more adaptationist accounts of human culture fall short. This point is illustrated by the fact that the superstimulus-concept appears to play an important role in certain evolutionary psychologically inspired (by-product) explanations of religion and religious behavior (Boyer 2001, Sperber \& Hirschfeld 2006). For instance, after having stated that "[h] umans habitually 'fool' their own innate releasing programs", Scott Atran goes on to hypothesize that "supernatural agents are readily conjured up perhaps because natural selection has tripwired cognitive schema for agency detection in the face of uncertainty" (Atran 2006, 306 and 307). 
In short, the superstimulus-concept often appears to be invoked in a rather casual, perhaps even almost obligatory fashion, without much concern for its further theoretical development. An exception to this would certainly be Dan Sperber's well-known conceptual elaboration of the notions of different domains of psychological modules (Sperber 1996, Sperber \& Hirschfeld 2006). Closely related to the aforementioned evolutionary psychological mismatch hypothesis, or at least to what we hold to be its third sub-claim - some objects or events in our cultural environment resemble objects or events from our ancestors' environment, triggering reactions that evolved as adaptive responses to the Pleistocene objects and events - he suggests that a module's proper domain be distinguished from its actual (and cultural) domain(s). Sperber's views on the relations between these different domains provide us with a first model of how to make sense of superstimuli in the context of human behavior. In the following section, we discuss and elaborate several of such models, including Sperber's.

\section{Different approaches to supernormal stimulus selection}

There appear to be different ways in which the occurrence of supernormal stimulation can be conceptualized. Most of the evolutionary psychological literature addressed above makes use of some version of Lorenz and Tinbergen's largely embryonic conceptual framework and its accompanying classical examples and experiments. The early ethological concept, "superstimulus", was of course intimately connected to other erstwhile core ethological notions, such as the innate releasing mechanism, sign stimuli and the fixed action pattern notions which nowadays have, for the most part, been discarded by ethologists, or at any rate appear to go unused by most of them.

This is all the more reason for concern given that, as stated by Richard Burkhardt, perhaps ethology's foremost historian, “[o]ne striking feature of ethology's history is that the ideas that constituted the conceptual core of classical ethology were relatively short lived" (Burkhardt 2005, 15). Likewise, already twenty years ago, Bateson and Klopfer took "the view that ethology as a coherent body of theory ceased to exist in the 1950s. [...] One by one the concepts and theories succumbed to critical analysis and, by the beginning of the 1960s, any vestiges of common belief in an ethological theory of behavior had disappeared" (Bateson \& Klopfer 1989, vi). Even so, and notwithstanding Tinbergen's own cautious remarks that "[t]he full significance of the phenomenon of 'supernormal' sign stimuli is not yet clear. A 
closer study might well be worthwhile" (Tinbergen 1951, 46), a lot of evolutionary minded human behavioral scientists nowadays seem content to apply these ideas, implicitly or explicitly, in their work. This seems true even for Sperber, even though, as we shall see, his conceptual framework is by far the best explicated one.

The purpose of the present section is to reconnect the discussion of superstimuli in humans with more recent, mainly theoretical, ethological literature on stimulus selection and supernormal stimulation. The problem of supernormal stimuli may be approached as pertaining to the more general problem of "generalization" or, in the wordings of Ghirlanda and Enquist $(2003,15)$, the question of "how animals respond to sets of stimuli including familiar and novel stimuli", or perhaps more specifically, "how an animal will react to novel stimuli that are somewhat different from familiar ones, to which the animal's reactions are known" (Ghirlanda 2002, 389). It is interesting to note that these authors stress the relative neglect of this topic in both recent ethology and psychology, and connect it explicitly to the phenomenon of supernormal stimulation (Ghirlanda 2002; Ghirlanda \& Enquist 1999). Taking inspiration from this literature, we suggest the concept of biases in the psychological makeup of the receiver of a stimulus or combination of stimuli, and their possible exploitation, as an overarching category within which to address superstimuli. Within this overarching category of biases, a first distinction can be introduced between functional and non-functional biases. As we will see, this will allow for a reconceptualization of Sperber's formulation of (supernormal) stimulus selection in terms of domain-specificity and modularity.

Functional biases

The very notion of a module coming equipped with its proper domain entails that said module evolved as an adaptation to certain domain-specific problems. The proper domain of a cognitive module is "all the information that it is the module's biological function to process" (Sperber 1996, 136, cf., also Sperber \& Hirschfeld 2006, 157 for a recent and somewhat different articulation). For instance, the proper domain of a face recognition module would be natural faces. Cultural artifacts, such as masks, would belong to the actual domain of this module if they meet the module's input conditions. However, masks would not belong to its proper domain, even though they would stimulate the mechanism, because this mechanism was not designed by natural selection to recognize masks. The relationships between a 
module's proper and actual domain that we are interested in here can then take on at least one of the two following forms: mismatches between domains, and mismatches of domains.

\section{Mismatches between domains, or stimulus variations along rearrangement dimensions}

Novel stimulus configurations satisfying the input conditions of a module, hence pertaining to its actual domain, do not necessarily belong to the proper domain of the module. Indeed, this often appears to be the case. Religious representations, or more specifically cases of supernaturalism, are often treated as paradigmatic of this type of supernormal stimulation. According to Sperber and Hirschfeld (2006, 164):

Representations of supernatural beings [...] spread and stabilize in different cultures because they act for one or several cognitive modules as superstimuli. Unlike other superstimuli, which have some features exaggerated while essential features are maintained, these cultural superstimuli typically combine exaggerated and paradoxical features with ordinary and essential ones. One way they may be paradoxical is in falling simultaneously in the actual domain of two different modules.

Rather than using the distinction between proper and actual domains of modules, Ghirlanda and Enquist introduced, admittedly in a markedly different context, the notion of dimensions along which stimuli can vary (Ghirlanda \& Enquist 2003). This notion of stimulus dimensions lends itself to expressing ideas quite similar to the ones envisaged by Sperber and Hirschfeld, even though neither author pair refers to the other in their respective work on the subject of supernormal stimulation. Nor, for that matter, has this connection, to our knowledge, been made by other authors working in this area.

The basic dichotomy presented by Enquist and Ghirlanda involves, on one hand, (qualitative) variations along rearrangement dimensions (such as object shape, orientation or location), taking into account how an amount of stimulation is distributed among the receptors of the sense organs involved, and, on the other hand, (quantitative) variations along intensity dimensions (such as intensity of sound, light or chemical concentration), referring to the total activation of these receptors.

Mismatches between the proper and actual domains of modules could be interpreted as often involving (mere) variations along rearrangement dimensions of the stimuli, causing some 
input to meet some module's actual input conditions. Ghirlanda and Enquist's research suggests that, while response biases or stronger responses towards other stimuli than the originally existing ones do occur, this is far from always being the case. Phenomena like supernaturalism may involve both mismatches between proper and actual domains (in Sperber and Hirschfeld's terminology) and variations along rearrangement dimensions of the relevant stimuli (in Ghirlanda and Enquist's terminology). This is not to say, however, that they are necessarily appropriately called superstimuli, since actual response biases are relatively rarer along such rearrangement dimensions than they are in other cases, as, for example, we shall show with the case treated in the next subsection. Moreover, as shown in an obviously highly idealized fashion in the figure below, Ghirlanda and Enquist's meta-analysis of experimental data suggests that responses to stimuli varying along rearrangement dimensions are usually described better by - peaked - bell-shaped or Gaussian curves.

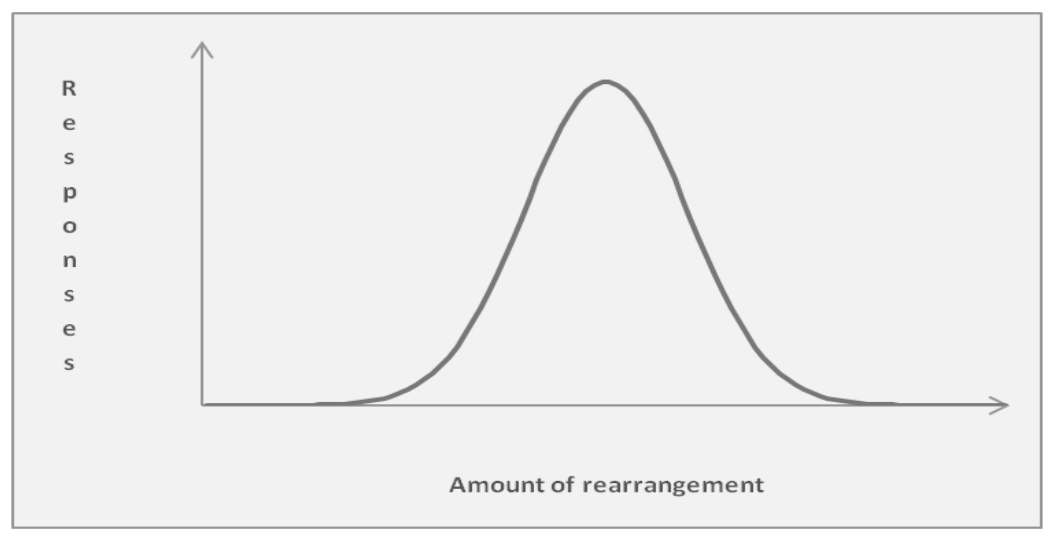

Fig. 1 Responses to stimuli as a function of stimulus variations along rearrangement dimensions

Possible evolutionary reasons for this relative lack of response biases when dealing with stimulus variations along rearrangement dimensions, as well as for the less distinct nature of actually occurring response biases in these cases, could include the following. It is for instance unclear what open-endedness could possibly be referring to for these types of stimulus variations. Intuitively, it seems to make very little sense for an organism to evolve a bias for the most shuffled stimulus configuration in a certain domain, given that there will rarely be anything resembling a straightforward positive correlation between the amount of stimulus rearrangement and the adaptive value of preferentially or more forcefully reacting to the rearranged stimulus variant. Generally speaking, there appears to be little reason why the mere shuffling of the various elements constituting a complex stimulus configuration would 
inexorably lead to preferences, exaggerated or not, for thus shuffled stimulus variants. At the very least, as we will see in the next subsection, there are better reasons to believe why such preferences are more likely to arise when confronted with mismatches of domains, or perhaps better, stimulus variations along intensity dimensions.

\section{Mismatches of domains, or stimulus variations along intensity dimensions}

Another case, to some extent perhaps also envisaged by Sperber (2007), would consist in the fact that stimuli, even though pertaining to both the proper and the actual domains of a given module, are more readily available or stronger in our novel environments than they were under the ancestral conditions in which this module evolved. Such, more readily available or stronger, stimuli often tend to elicit stronger responses from their receivers, potentially leading to maladaptive results. One of the most oft-cited examples of superstimuli with regard to human behavior concerns the dangerous human preponderance towards sugar and fat (see, e.g., Symons 1979). Much of this more recent literature echoes Lorenz's views on the subject:

The art of cooking, the competition of chefs catering for the most sophisticated gourmets, long ago inspired the invention of supernormal food stuffs, much to the detriment of civilized humanity. For our paleolithic ancestors, hungry as they were much of the time, it was certainly sound strategy to follow the instructions of IRMs telling them what foods to choose: they should contain as much fat as possible, as much sugar as possible, and as little roughage as possible. Being "open on one side," these key stimuli led to an extremely unhealthy preference for supernormal objects. [...] Even the most complete insight into the workings of our IRMs does not make it easy to avoid suicide by overeating. (Lorenz 1981, 165-166) ${ }^{2}$

This second category of biases would seem to correspond more closely to Ghirlanda and Enquist's second main stimulus dimension, involving variation in the intensity of stimulation. While it is acknowledged that the distinction between intensity and non-intensity or rearrangement dimensions is in itself insufficient to cover each and every instance of variation between often-complex stimulus configurations, it does seem to offer some advantages over

\footnotetext{
${ }^{2}$ We would like to acknowledge here that the case of modern day humans over-eating sugary or fatty foods is not necessarily the best example of supernormal stimulation in action. The reason for over-eating these foods may very well lie in the mechanisms that control appetite, rather than in them being preferred because they are sweeter or fatter. Nevertheless, given its prevalence in the literature, we have chosen to retain this example.
} 
other approaches to stimulus generalization. Whereas Sperber's largely implicit classification of superstimuli provides little guidance as to when to expect them to occur more prominently, Ghirlanda and Enquist's taxonomy allows us to predict stronger response biases when confronted with stimuli whose supernormal character is due do their intensity. Moreover, according to the ethological and psychological literature reviewed by Ghirlanda and Enquist, many intensity generalization gradients are monotonic up to a certain threshold (rather than peaked, as we saw to be the case for rearrangement gradients). Figure 2 illustrates how responses to these types of stimulus variations often tend to increase linearly (up to a certain threshold) with the intensity of the stimulation involved.

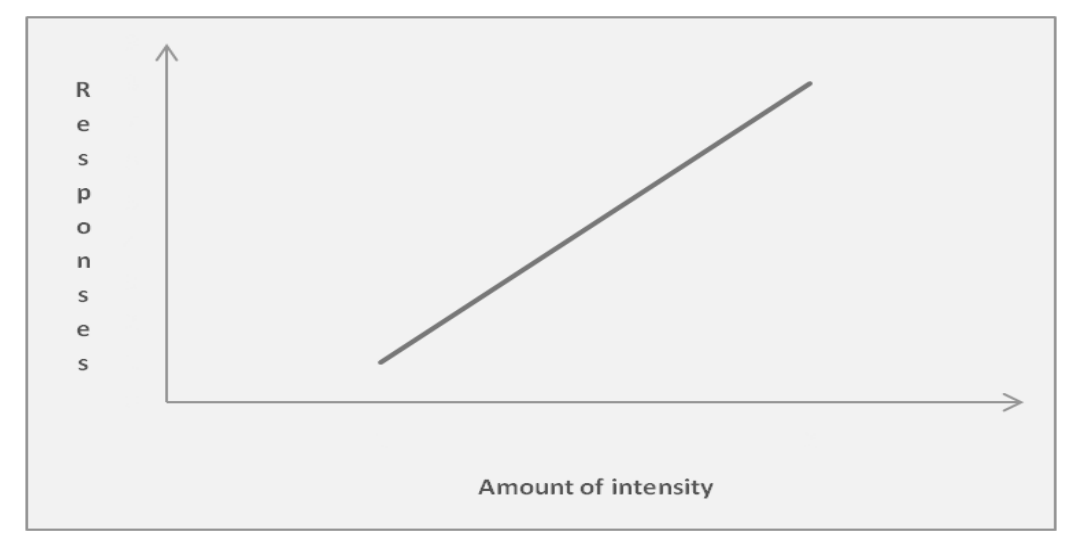

Fig. 2 Responses to stimuli as a function of stimulus variations along intensity dimensions

This dovetails quite nicely with Lorenz's intuitions about the open-ended nature of certain stimuli in general, but has the advantage of being more specific as to why some types of stimulus variants tend to exhibit this characteristic more often than others, or are, so to speak, more open to open-endedness. Indeed, Ghirlanda and Enquist's approach clearly indicates which types of stimulus variations are prone to leading to response biases. Again, we can speculate about the evolutionary origins of such response biases. What is perhaps the most intuitively appealing possible explanation refers mainly to the absence of counter-indications against these biases evolving. For example, according to Manning and Dawkins, rules of thumb like approach the largest female are adequate and evolve easily simply because false positives are rare (Manning \& Dawkins 1993).

Obviously, quite a few of the phenomena that are actually labeled as superstimuli will involve variations along both intensity and rearrangement dimensions, making it more difficult to 
assess the usefulness of Ghirlanda and Enquist's framework in these cases. Nevertheless, some stimulus variations involving changes in both intensity and arrangement, such as the aforementioned variations in size, appear to show response biases at least in some respects similar to those obtained along intensity dimensions (Ghirlanda \& Enquist 2003).

\section{Non-functional biases}

Our final category involves adaptively neutral, or non-functional biases. In their recent reaction to Richerson and Boyd's Not by Genes Alone, Sperber and Claidière offer a simple example for consideration when addressing what Richerson and Boyd call "content-based bias" in cultural evolution. When people are simultaneously exposed to two equally appreciated jokes, the first of which, however, is harder to remember than the other, they plausibly argue that the latter is more likely to spread and become stabilized in the population (Sperber \& Claidière 2008, 286). In the context that concerns us here, this case could also be interpreted as illustrating the workings of a neutral memory bias. Given certain general properties of our neuronal circuitry, not directly related to the type of information at hand, some information will be more easily memorized than other information. ${ }^{3}$

As such, we believe that this example bears some obvious resemblance to Enquist and Arak's by-product explanation for the existence of certain types of biases (Arak \& Enquist 1993; Enquist \& Arak 1998). While their neural network modeling results have been criticized by other behavioral ecologists (cf., for example, Dawkins \& Guilford 1995, and Endler \& Basolo 1998 for a review), it remains plausible that at least some instances of supernormal stimulus generalization result from general biases of the sensory system. That is, biases not specifically designed by natural selection to solve any (domain-)specific non-sensory adaptive problem. According to Enquist and his collaborators, these general properties can nonetheless drive the evolution of signal design towards what they call "hidden preferences", among which, again, there may be a - hidden - preference for exaggerated stimuli. This last category of superstimuli thus stands in no direct relationship with any pre-existing adapted or adaptive bias. This might explain why the evolutionary psychological literature on superstimuli

\footnotetext{
${ }^{3}$ This is obviously not to state that memory as such serves no function; it only means that such function is not, or at the very least not necessarily, related to, for example, the adaptive significance of humour. Put differently, "non-functional" refers to the specific problem under scrutiny, not to the absence of any function at all.
} 
neglects it. However, Enquist et al.'s theoretical framework leaves ample room for such a category.

To conclude this section, we argue that the incorporation of recent ethological theorizing on the phenomena of superstimuli and bias exploitation can lead to a considerable refinement of the existing conceptual framework of supernormal stimulation in humans. Sperber and his colleagues (Hirschfeld and Atran) may have aimed at a similar refinement when they tried to connect supernormal stimuli with some key notions of evolutionary psychology's massive modularity framework, such as domain specificity, proper domain, and actual domain. However, we find our approach to be preferable for several reasons. First, the domainspecificity of (all) our mental processing systems is far from certain (Currie \& Sterelny 2000). Second, the distinction between the actual and the proper domain of a function is explicitly inspired by the distinction between actual (or weak) and proper functions, elaborated by Millikan, Neander and other etiological theorists of biological function. However, this etiological theory has many problematic features (Wouters 2005), some of which have a bearing on the distinction at stake here. This is particularly true for the problem of functional indeterminacy (or, more precisely, the problem of the extensionality of natural selection): only denying (or neglecting) natural selection's extensionality can result in the distinction between the actual and the proper domain of a module. The validity of Sperber's claim that the proper domain of our face-recognition module is to recognize natural faces (and not masks) depends on the assumption that the only possible description of the module is that of a 'face-recognizing device'. Following Fodor (1996), we believe that this assumption is at least partially unwarranted, as, according to Sperber, the content "natural faces" was co-extensional with the content "face-like objects such as masks and natural faces" in the environment in which humans evolved. Third, Sperber's proposal tends to obfuscate the prefix "super" in "superstimulus". In the evolutionary psychological(ly) inspired literature, the superstimulusconcept tends to be used indiscriminately to designate the workings of the three categories of biases we distinguished in this section. Our analysis, however, suggests a more restricted usage of the term superstimulus which seems more in line with its original formulation in the ethological literature: restricted to cases involving actual response biases. Fourth, trying to bring the discussion on superstimuli in the human evolutionary social sciences under the general heading of biases and their possible exploitation would probably extend more easily into the existing literature on the role and significance of (other) psychological biases in cultural evolution (see, e.g., Sperber \& Claidière 2008; Richerson \& Boyd 2005). However, 
even though we believe our refined conceptual framework offers significant advantages, it is not without problems of its own. As we explain in the next section, most of these problems are related to the aforementioned phenomenon of cultural evolution.

\section{The problem with humans}

Humans are a cultural species. This raises the question how being a cultural species is likely to affect the exploitation of our biases. Some evolutionary psychologists (e.g., Pinker 1997) suggest that, thanks to our general and social intelligence, language, our capacity to plan, and our imaginative capacities, humans are simply better than other species in manipulating conspecifics (Mithen 1996, Carruthers 2006). At some point in the course of our hominid lineage, these basic abilities - abilities we do not share with most of our primate relatives may have been culturally exapted to create misleading cues of survival and reproductive success that nonetheless meet the input conditions of the mind's processing systems.

We do not want to question the claim that humans are particularly good at creating superstimuli. In fact, Carruthers's convincing evolutionary account of our creative cognition (Carruthers 2006) provides an important building block towards an encompassing theory of bias exploitation in humans and human culture. Nevertheless, his evolutionary account of human creativity, convincing as it may be, cannot be the encompassing theory itself. Apart from our (supposedly) spectacular capacity to exploit the biases of conspecifics, there are many other differences in bias exploitation between our species and other species. Most of these differences have to do with culture. In this section, we will explore these differences and explicate why they put important constraints on the use of the superstimulus-concept, be it a coarse or a nuanced one, in the evolutionary social sciences.

Strategic and non-strategic exploitation

Much of the research and theorizing about the exploitation of sensory and cognitive biases is done in the context of sexual selection. ${ }^{4}$ In the sensory bias model of sexual selection, a

\footnotetext{
${ }^{4}$ It is interesting to note that Lorenz apparently disliked the theory of sexual selection, and saw his concept of the releaser as a valid alternative. Or, in Burkhardt's $(2005,169)$ wording: "Finding the idea of sexual selection repugnant, he was happy to account for the majority of secondary sexual characters in other terms. As he saw it, the majority of conspicuous structures, colors, sounds, and behavior patterns in animals served as releasers of
} 
female preference for a male trait is seen as the result of biases in the sensory system of the female, a system that existed prior to the evolution of the preferred male trait (Andersson 1994, Ryan \& Rand 1990). Most studies done on sensory exploitation outside the sexual selection context concern interspecies deception by means of signal imitation (Dawkins \& Krebs 1979). The begging calls of the cuckoo chick, for example, imitate the calls of an entire brood of the host species, thus eliciting (or exploiting) the childcare behavior of the host parents (Davies et al. 1998). Deception within and between species has in common that one party is duped, while the other party benefits from exploiting the bias. Although some have argued that a dupe is not needed for exploitation (e.g., Dawkins \& Guilford 1996), there seems to be no exploitation at all if no one profits from it.

In short, bias exploitation in animals occurs most often in a strategic context. But, is this also true for "bias exploitation" in humans? Is the human receiver always duped? And, does the human sender always benefit?

\section{Who benefits?}

The above-mentioned example of cooking and sugar overconsumption illustrates that it may remain unclear who benefits from an alleged deception. To tackle this problem, one might initially suggest that it is obviously the cook (or the candy-producer) who benefits. This solution, however, is not completely satisfactory for several reasons. Foremost, the cook's cooking seems more beneficial to his wallet than his reproductive success. But, in the animal ecology literature on sensory exploitation, only benefits in terms of reproductive fitness count as real benefits. Furthermore, the cook will perhaps often prepare exquisite, but not necessarily nutritious, dishes for himself. His benefit then is pleasure, but this short-term benefit is essentially an evolutionary cost.

Of course, this difference between sensory exploitation in humans and sensory exploitation in other animals should not be exaggerated. Self-exploitation through sensory biases via extracorporal artifacts is not a unique human behavior. Male fiddler crabs are themselves attracted by the mounds of sand they build to attract females (Ribeiro et al. 2006). Inversely, humans do use sensory exploitation in strategic contexts: make-up and high heels are often seen as 
superstimuli used by women to attract men (Frank 2007). Still, we think it is important to stress that very often the only beneficiary of sensory exploitation is the cultural artifact or the cultural practice itself. This is rarely seen in nonhuman animals. It seems quite likely that this difference is due to the difference between cultural and natural functions (Richerson \& Boyd 2005, 165).

\section{Who is the dupe?}

When evolutionary psychologists and other evolutionary social scientists write about superstimuli, the "dupe" is always easy to identify: we, humans, are supposed to be misled by cultural artifacts, because these artifacts are or contain exaggerated sensory signals. However, one should be cautious about underwriting this claim for at least three important reasons. The first problem is that many of the cultural products labeled as detrimental superstimuli are actually nothing of the sort, but rather stimuli that enhance the receiver's fitness. Carroll, for example, criticizes Pinker and others (like Ramachandran) who see art as a complex of superstimuli. According to Carroll, art is most likely an adaptation:

If we compare the effects of music with those of recreational drugs, we can begin to understand the mistaken direction Pinker's theory has taken. Drugs are disorienting and demoralizing. If young people use them habitually, they become incapable of adapting to the demands of a complex environment. Music has no such deleterious effect. More importantly, it seems very likely that people raised with no exposure to music, art, or literature would be psychologically and emotionally stunted, that they would be only marginally capable of developing in normal ways. They would probably have great difficulty learning to deal with their own emotions or to relate to other people with any sensitivity and flexibility. Their capacity for responding in creative ways to the demands of a complex and changing cultural environment would probably be severely impaired. (Carroll 1998, 481)

On this view, music, literature, and painting can still be exaggerated signals, but it would be a mistake to say that they exploit our biases. Actually, it is not inconceivable that they even have more beneficial results (for the receiver) than the natural stimuli for whose processing the biases were initially designed by natural or sexual selection. The open-endedness of such 
biases should then be thought of as an adaptive byproduct, and not as a maladaptive or functionally neutral one.

The second problem is that of the supposedly addictive character of real superstimuli. Are we really as vulnerable to bias exploitation as some evolutionary psychologists suggest? Or, has our evolution as a cultural species led to defenses against such forms of exploitation? As Carroll notes, art is not as addictive as heroin or even cheesecake, so it seems inappropriate to call literature "cheesecake for the mind". Yet, at the same time, one should not forget that heroin and cheesecake are not equally addictive for everyone. The psychological literature on addiction mentions two correlated factors to account for inter-individual differences. The first is variation in our biases. The second is variation in our capacity for self-control. This second factor entails that most humans are not simple pleasure machines. Yes, we do have "pleasure buttons" that can be pushed by artifacts, mental representations and natural stimuli, but our biases are seldom so exploited that we become prisoners of these pleasure buttons. This is illustrated by Robert Nozick's well-known thought experiment, dubbed "the experience machine" (Nozick 1974). The experience machine, that Nozick asks us to imagine, can give us whatever pleasurable experiences we could possibly want. "Superduper neuropsychologists" have figured out a way to stimulate a person's brain in order to induce any pleasurable experience they may desire. We would not be able to tell that these experiences were not caused by the real thing. Then, he asks us, if we were given the choice, would we like to plug in or not? Most people, says Nozick, would be unwilling to plug in, because we also care about living in contact with reality. Pleasure (or the absence of suffering) is not the only thing that matters to us. As a matter of fact, this reluctance in relation to the experience machine is not strange. If we are truly a cultural species, chances are that cultural superstimuli have been around for quite a long time, probably long enough for gene-culture co-evolution to have resulted in a mechanism to counter their detrimental effects. In other words, it is quite likely that we have evolved defenses against bias exploitation by culture. We are not immune to temptation, but our power to resist this temptation is probably stronger than that of other animals (Logue 1988). Moreover, such selfcontrol is not very domain-specific (Gailliot \& Baumeister 2007). Because we are the single species that can form beliefs about our own intentional states, the human mind has the ability to regulate itself, unlike the minds of other animals (McGeer \& Pettit 2002). This means that most modern temptations are probably well within the range of self-control. One can conclude 
that humans are less susceptible to bias exploitation than most evolutionary psychologists seem to think. Or, as Boyd and Richerson put it:

[E]nvironments outside the range in which a species has evolved are quite likely to result in a miscellany of breakdowns and manipulations. Humans are not the best candidates to exemplify such breakdowns, because we are a species that is superbly adaptable to variable environments, as our explosive success during the Holocene testifies. (Richerson \& Boyd 2005, 189)

A third, somewhat related, problem has to do with the fact that humans often actively choose to pursue the exploitation of their biases. Most people have long and short-term goals. Among those short-term goals, pleasure normally ranks high. It goes without saying that we sometimes prefer short-term superstimulation to happiness in the long-term. One could claim that, in such cases, we succumb to the temptation presented by the superstimuli. However, unlike other animals, humans sometimes also choose to be fooled or manipulated by cultural superstimuli. We go to the theatre and the movies, knowing that what we are going to see is not real. Superstimulus enthusiasts would perhaps argue that this underscores their point: superstimuli are tempting to the point of being irresistible, even despite our knowing, in some cases, that they are not real. However, we believe that this is simply incorrect. What it actually shows is that we sometimes decide that it does not matter to us all that much that we are being manipulated. As our self-regulating mind can intentionally seek to discover what is real and what is not, a desire for fun can lead it intentionally not to pursue such questions (McGeer \& Pettit 2002). Something like intentional self-deception is unlikely to occur in other animals, but its occurrence in humans is undisputed (Mele 1987). Gombrich $(2000,87)$ correctly notes that humans "are not simple slot machines which begin to tick when coins are dropped into us, for, unlike the stickleback, we have what psychoanalysts call an 'ego' which tests reality and shapes the impulses from the id. And so we can remain in control while we half-surrendered to counterfeit coins, to symbols and substitutes." Obviously, we are well aware of the fact that psychological experiments find that we are less in control than we think we are (Saad 2007). But, that does not detract from the point that, despite considerable interindividual variation, most of us are to a large degree in control of what we do, even if what we do is not in our long-term (evolutionary and other) interests (Baumeister et al. 2007). 
Innate and learned biases

Traditionally, the ethological literature attached a great deal of importance to purported differences between innate and learned behavioral patterns. Lorenz, for instance, writes:

[T] he IRM cannot do what is so easily done by our learned gestalt perception, that is, respond selectively to complex qualities. It is an extremely reliable rule of thumb that an IRM can be assumed to be at work whenever an organism is "taken in" by a very simple dummy or model. Conversely, if the attempt to elicit a certain response by a dummy fails, and it proves necessary to simulate a biologically relevant stimulus situation in all its details in order to release a response, or if even this proves to be impossible, the assumption is justified that the organism has learned to respond to a complex quality. (Lorenz 1981, 171) ${ }^{5}$

This quote clearly shows that Lorenz' account of innateness refers to a cluster of several properties. On his view, IRMs are innate because they are (a) not learned, and (b) highly environmentally canalized. As others have argued (e.g. Bateson \& Mameli 2007, Mameli 2008), the inference from highly environmentally canalized to not learned is not always warranted. Apart from this philosophical problem, however, there is little or no theoretical and empirical support for the idea that stronger responses to superstimuli are generally the effect of non-learned recognition system biases. Ghirlanda and Enquist, for example, do not appear to find significant differences between the generalization of genetically inherited and individually learned behavior in the available data. More specifically, they find no support for the (early) ethological claim that biases in genetically inherited behavior are open-ended, whereas biases in learned behavior are limited (Ghirlanda \& Enquist 2003).

This suggests that the occurrence of superstimuli does not necessarily prove that the phenomenon under consideration cannot possibly be the result of learning, contrary to what has been and sometimes still is suggested. The fact that superstimuli exist in a given context

\footnotetext{
${ }^{5}$ This is followed somewhat further on page 172 by the observation that "[i]t is perfectly conceivable that there might be releasers, or stimulus-emitting organizations, the signals of which are not addressed to an IRM but are received by learned perceptions, as are the color patterns of our flags. The functions of perception can certainly cause the production of signals catering to the properties just described. While there is an abundance of manmade signals whose properties are clearly dictated by the Prägnanztendenz of human gestalt perception, we know only a few examples of phylogenetically programmed stimulus emitters without a corresponding IRM, in other words, a releaser the response to which must be learned."
} 
appears to be compatible with the behavior in question being individually or socio-culturally learned.

Mameli calls the incompatibility of learning and innateness 'the minimal condition' which any account of innateness must meet (Mameli 2008, 721). But in the discussion on bias exploitation, the main focus of the nativist debate is less on this minimal condition, but rather on the issue whether or not the bias is (a) a genetically selected adaptation, and (b) a domainspecific bias. Unfortunately, there is not much space to discuss this issue here in full detail. But at least it is important to note that much of the nativist debate in the context of human bias exploitation is actually about the adaptiveness and domain specificity of learning mechanisms and cultural capacities (Bateson \& Mameli 2007, 827). Returning to the conceptual framework elaborated by Sperber, who readily acknowledges that "most innate human modules are learning modules" (Sperber \& Hirschfeld 2006, 157), a distinction could be made between source-based psychological biases and content-based psychological biases (Sperber \& Claidière 2008). Being "effects of the cognitive mechanisms that construct a mental representation on the basis of informational input" (Sperber \& Claidière 2008, 288), content-based biases could very well, depending on their relative open-endedness, be prone to supernormal stimulation. However, the ways in which such content-based biases interfere with source-based psychological biases (e.g., Boyd and Richerson's conformity bias and prestige bias), plausibly leading to different cultural evolutionary outcomes, depending on their relative importance in any given case, is still very much open to debate (compare, for example, Henrich et al. 2008 and Richerson \& Boyd 2008 with Sperber \& Claidière 2008). Following up on our earlier example of the cook, and paraphrasing somewhat Henrich et al. (2008, 127), it could, for instance, be argued that the fact that a given novel recipe corresponds well with attracting innate taste preferences and/or learned local cuisine styles will by no means necessarily guarantee it spreading if, for whatever reason, the cooks producing this particular recipe are unlikely to be selected as cultural models. Moreover, the source-based psychological biases' vulnerability to superstimuli merits further investigation. Would, for example, the more extreme cultural role models generate more intense response biases under certain circumstances? Or, put differently, to what extent are biases like these themselves merely directional, or conversely, rather more open-ended?

Finally, content-based biases are also likely to exhibit different degrees of learnedness. Thus, what Sperber and his collaborators refer to as cultural attractors are equally likely to be 
subject to these differences. Not only can concrete factors of attraction be of a psychological or ecological character (Sperber 1996; Sperber \& Claidière 2008), they may also quite often rely on learning and culturally transmitted preferences, allowing for changes of attractors on historical timescales (Claidière \& Sperber 2007). Placing the discussion of superstimuli within this broader framework is perhaps capable of generating a very different, more complete picture than the one traditionally espoused.

\section{Conclusion: Are we amusing ourselves to death?}

It may be the case that we and other, more or less civilized aliens appear prone to driving ourselves to extinction. But, even then, this would not have to mean that the Fermi paradox can be resolved simply by appealing to the superstimulus-theory. As we have argued, several specificities common to profoundly cultural species would likely preclude them from succumbing entirely to the temptations of supernormal stimulation. Obviously, that is not to say that humans are completely immunized against the exploitation of their biases. Although we have argued that human culture and our evolved cultural capacities probably tend to soften or even counter the fitness-detrimental effects of such exploitation, we nonetheless acknowledge that culture sometimes has the opposite effect. Why would this be the case? Is it because cultural species are simply better at constructing superstimuli than other animals? Surely, this seems to be part of the answer. However, we will conclude this paper by arguing that fitness-detrimental bias exploitation in our species might also be partially explained by the peculiarities of cultural transmission itself.

Our - now globalized - Western culture produces individuals who would rather pursue pleasure than maximize their fitness. But this is both historically and cross-culturally quite exceptional. In most cultures most people behave in ways that tend to maximize fitness. One can safely assume that they are not amusing themselves to extinction. This raises the question of why seeking pleasure is (much) more valued in Western culture than in other cultures. What happened to the cultural transmission of norms, such that pleasure-seeking has become more value than vice?

In a series of papers, Newson and Richerson have argued that many traditional communities maintain fitness-maximizing norms because a large proportion of social interaction is between 
kin. Because kin have an interest in encouraging each other towards higher fitness, their implicit and explicit communications are more likely to favor behavior that strongly correlates with high fitness (Newson et al. 2007, Newson \& Richerson 2009). Conversely, a decrease in the frequency of contact between kin leads to a progressive relaxation of norms that encourage fitness-maximizing behavior. According to Newson and Richerson, this explains the demographic transition and a series of other changes in reproductive behavior (Newson \& Richerson 2009). Following Durkheim and other social scientists, they believe that with industrialization, institutions such as businesses, schools and political systems, begin to assume roles and responsibilities that were once the province of the family.

This line of reasoning can easily be applied to cultural differences in bias exploitation. While individuals in pre-industrial societies conform to the family values that tend to maximize fitness, individuals in industrialized societies are more inclined (a) to develop strong preferences of their own, and (b) to act according to those individual preferences. In industrialized societies, the fitness-detrimental exploitation of evolved and other biases will probably be stronger, not solely because of the large-scale production of superstimuli and the rise of a consumption culture (cf. Enquist et al. 2002), but also because industrialization reduces the social control of kin. Perhaps somewhat paradoxically, this shows that an evolutionary psychological approach, focusing on individual preferences and superstimuli, may have more predictive and explanatory value in our contemporary industrialized culture precisely because of modern, culturally induced changes in cultural transmission. 


\section{References}

Andersson M (1994) Sexual Selection. Princeton University Press, Princeton Arak A, Enquist M (1993) Hidden preferences and the evolution of signals. Phil. Trans. R. Soc. Lond. B 340:207-213

Atran S (2006) Religion's Innate Origins and Evolutionary Background. In: Carruthers P, Laurence S, Stich S (eds) The Innate Mind: Culture and Cognition. Oxford University Press, Oxford, pp 302-317 Bateson P, Klopfer P (1989) Preface. In: Bateson P, Klopfer P (eds) Perspectives in Ethology Volume 8: Whither Ethology. Plenum, London/New York, pp v-viii

Bateson P, Mameli M (2007) The Innate and the Acquired: Useful Clusters or a Residual Distinction From Folk Biology? Developmental Psychobiology 49:818-831

Baumeister R, Vohs K, DeWall C, Zhang L (2007) How emotion shapes behavior: Feedback, anticipation, and reflection, rather than direct causation. Personality and Social Psychology Review 11:167-203

Behrens R, Whitson P (1976) Mimicry, Metaphor, and Mistake. Journal of Aesthetic Education 10:4560

Blanchette I (2006) Snakes, spiders, guns, and syringes: How specific are evolutionary constraints on the detection of threatening stimuli? Quarterly Journal of Experimental Psychology 59:1484-1504

Boyer P (2001) Religion explained: the evolutionary foundations of religious belief. Random House, London

Burkhardt R (2005) Patterns of Behavior: Konrad Lorenz, Niko Tinbergen, and the Founding of Ethology. University of Chicago Press, Chicago

Carroll J (1998) Steven Pinker's Cheesecake for the Mind. Philosophy and Literature 22:478-485

Carruthers P (2006) The Architecture of the Mind: massive modularity and the flexibility of thought. Oxford University Press, Oxford

Claidière N, Sperber D (2007) The role of attraction in cultural evolution. Journal of Cognition and Culture 7:89-111

Coss R (1968) The ethological command in art. Leonardo 1:273-287

Currie G, Sterelny K (2000) How to think about the Modularity of Mind-Reading. The Philosophical Quarterly 50:145-160

Davies N, Kilner R, Noble D (1998) Nestling cuckoos, Cuculus canorus, exploit hosts with begging calls that mimic a brood. Proceedings of the Royal Society London, B 265:673-678

Dawkins M, Guilford T (1995) An exaggerated preference for simple neural network models of signal evolution? Proceedings of the Royal Society London, B 261:357-360

Dawkins M, Guilford T (1996) Sensory bias and the adaptiveness of female choice. The American Naturalist 148:937-942

Dawkins R, Krebs J (1978) Animal signals: information or manipulation. In: Krebs JR, Davies NB (eds) Behavioural Ecology: An Evolutionary Approach. Blackwell Scientific Publications, Oxford, pp 282-309

Dawkins R, Krebs J (1979) Arms races between and within species. Proceedings of the Royal Society London, B 205:489-511

Endler JA, Basolo AL (1998) Sensory ecology, receiver biases and sexual selection. Trends in Ecology and Evolution 13:415-420

Engelmann F (1970) The Physiology of Insect Reproduction. New York: Pergamon Press

Enquist M, Arak A (1998) Neural Representation and the Evolution of Signal Form. In: Dukas R (ed) Cognitive Ethology: The Evolutionary Ecology of Information Processing and Decision Making. University of Chicago Press, Chicago, pp 21-87

Enquist M, Arak A, Ghirlanda S, Wachtmeister C-A (2002) Spectacular phenomena and limits to rationality in genetic and cultural evolution. Phil. Trans. R. Soc. Lond. B 357:1585-1594

Fodor J (1996) Deconstructing Dennett's Darwin. Mind and Language 11:246-262

Frank R (2007). The Economic Naturalist: In Search of Solutions to Everyday Enigmas. Basic Books, New York 
Gailliot M, Baumeister R (2007) The Physiology of Willpower: Linking Blood Glucose to SelfControl. Personality and Social Psychology Review 11:303-323

Ghirlanda S (2002) Intensity Generalization: Physiology and Modelling of a Neglected Topic. Journal of Theoretical Biology 214:389-404

Ghirlanda S, Enquist M (2003) A century of generalization. Animal Behaviour 66:15-36

Ghirlanda S, Enquist M (1999) The geometry of stimulus control. Animal Behaviour 58:695-706

Gombrich EH (2000) Art and Illusion: a study in the psychology of pictorial representation. Princeton University Press, Princeton

Hailman J (1961) Why do gull chicks peck at visually contrasting spots: a suggestion concerning social learning of food-discrimination. The American Naturalist 95:245-247

Hansson H (1959) Effects of discrimination training on stimulus generalization. Journal of Experimental Psychology 58:321-333

Henrich J, Boyd R, Richerson P (2008) Five Misunderstandings About Cultural Evolution. Human Nature 19:119-137

Irons W (1998) Adaptively Relevant Environments Versus the Environment of Evolutionary Adaptedness. Evolutionary Anthropology 6: 194-204

Knobloch F (1995/2000) The Interpersonal Meaning of Music and Ethology. Available at: http://cogweb.ucla.edu/Abstracts/Knobloch_95.html

Kruuk H (2003) Nico's Nature: The Life of Nico Tinbergen and his Science of Animal Behaviour. Oxford University Press, Oxford

Lack D (1968) Ecological Adaptations for Breeding in Birds. Methuen, London

Logue A (1988) Research on self-control: an integrating framework. Behavioural and Brain Sciences 11:665-709

Lorenz K (1981) The Foundations of Ethology. Springer, New York

Magnus D (1958) Untersuchungen zur Bionomie und Ethologie des Kaisermantels. Zeitschrift für Tierpsychologie 15:398-426

Mameli M (2008) On innateness: the Clutter Hypothesis and the Cluster Hypothesis. The Journal of Philosophy 105:719-737

Manning E, Dawkins MS (1993) Introduction to animal behaviour. Cambridge: Cambridge University Press

McGeer V, Pettit P (2002) The Self-regulating Mind. Language and Communication 22:281-299

Mele A (1987) Recent Work on Self-Deception. American Philosophical Quarterly 24:1-17

Miller G (2006) Runaway consumerism explains the Fermi Paradox. Available at: http://www.edge.org/q2006/q06_9.html

Mithen S (1996) The Prehistory of the Mind: A Search for the Origins of Art, Religion and Science. Thames and Hudson, London/New York

Morris D (1969) The Human Zoo. Jonathan Cape, London

Newson L, Postmes T, Lea S, Webley P, Richerson P, McElreath R (2007) Influences on communication about reproduction: the cultural evolution of low fertility. Evolution and Human Behavior 28:199-210

Newson L, Richerson P (2009) Why do people become modern? A Darwinian mechanism. Population and Development Review 35:117-158

Nozick R (1974) Anarchy, state, and Utopia. Basic Books, New York

Öhman A, Mineka S (2001). Fear, phobias and preparedness: Toward an evolved module of fear and fear learning. Psychological Review 108:483-522

Pinker S (1997) How the Mind Works. Norton, New York

Pinker S (2003) The Blank Slate: the modern denial of human nature. Penguin Books, London

Ramachandran V (2004) Beauty or Brains. Science 305:779-780

Ribeiro P, Christy J, Rissanen R, Kim T (2006) Males are attracted by their own courtship signals.

Behavioral Ecology and Sociobiology 61:81-89

Richerson PJ, Boyd R (2005) Not by Genes Alone: How Culture Transformed Human Evolution. University of Chicago Press, Chicago

Richerson PJ, Boyd R (2008) Response to our critics. Biology \& Philosophy 23:301-315

Ryan M, Rand A (1990) The sensory basis of sexual selection for complex calls in the túngara frog, Physalaemus pustulosus (sexual selection for sensory exploitation). Evolution 44:305-314 
Saad G (2007) The evolutionary bases of consumption. Lawrence Erlbaum, Mahwah NJ

Sperber D (1996) Explaining Culture: A Naturalistic Approach. Blackwell, Oxford

Sperber D (2007) Seedless Grapes: Nature and Culture. In: Margolis E, Laurence S (eds) Creations of the Mind: Theories of Artifacts and Their Representation. Oxford University Press, Oxford, pp 124137

Sperber D, Claidière N (2008) Defining and explaining culture (comments on Richerson and Boyd, Not by genes alone). Biology \& Philosophy 23:283-292

Sperber D, Hirschfeld L (2006) Culture and Modularity. In: Carruthers P, Laurence S, Stich S (eds) The Innate Mind: Culture and Cognition. Oxford University Press, Oxford, pp 149-164

Stevens A, Price J (2000) Evolutionary Psychiatry: A New Beginning (2nd ed). Routledge, London Symons D (1979) The Evolution of Human Sexuality. Oxford: Oxford University Press.

Terrace H (1966) Stimulus control. In: Honig W (ed) Operant Behavior. Appleton Century Crofts, New York, pp 271-344

Tinbergen N (1948) Social releasers and the experimental method required for their study. Wilson Bulletin 60:6-52

Tinbergen N (1951) The Study of Instinct. Oxford University Press, London (quoted from 1989 reprint)

Tinbergen N, Perdeck AC (1950) On the stimulus situation releasing the begging response in the newly hatched Herring Gull chick (Larus argentatus Pont.). Behaviour 3:1-39

Wouters A (2005) The function debate in philosophy. Acta Biotheoretica 53:123-151

Zeki S (1999) Inner Vision: an exploration of art and the brain. Blackwell science, Cambridge 